\title{
Antihyperuricemic Effect of Dendropanax morbifera Leaf Extract in Rodent Models
}

\author{
Dongho Lee, ${ }^{1}$ Jin-Kyoung Kim, ${ }^{2,3}$ Yongjae Han, ${ }^{1}$ and Kwang Il Park $\mathbb{C}^{4}$ \\ ${ }^{1}$ Department of Anesthesiology and Pain Medicine, College of Medicine, Inje University, Gimhae 47392, Republic of Korea \\ ${ }^{2}$ Department of Preventive Dentistry, School of Dentistry Kyungpook National University, Daegu 41940, Republic of Korea \\ ${ }^{3}$ Department of Dental Hygiene, Daegu Health College, Daegu 41453, Republic of Korea \\ ${ }^{4}$ Institute of Animal Medicine and College of Veterinary Medicine, Gyeongsang National University, Jinju 52728, \\ Republic of Korea
}

Correspondence should be addressed to Kwang Il Park; kipark@gnu.ac.kr

Received 6 May 2021; Revised 8 July 2021; Accepted 16 July 2021; Published 23 July 2021

Academic Editor: Slim Smaoui

Copyright ( $\odot 2021$ Dongho Lee et al. This is an open access article distributed under the Creative Commons Attribution License, which permits unrestricted use, distribution, and reproduction in any medium, provided the original work is properly cited.

Dendropanax morbifera is a well-known traditional medicine used in China and Korea to treat intestinal disorders, urosis, diuresis, and chronic glomerulonephritis. Hyperuricemia is a metabolic disorder characterized by a high uric acid level in serum due to an imbalance between uric acid production and excretion and causes gout. Recently, the prevalence of hyperuricemia worldwide has been continuously increasing. Xanthine oxidase (XOD) inhibitors (allopurinol (ALP) and febuxostat) and uricosuric agents (benzbromarone and probenecid) are used to treat hyperuricemia clinically. However, because these drugs are poorly tolerated and cause side effects, such as kidney diseases, hepatotoxicity, gastrointestinal symptoms, and hypersensitivity syndrome, only a limited number of drugs are available. We investigated the antihyperuricemic effects of Dendropanax morbifera leaf ethanol extract (DMLE) and its underlying mechanisms of action through in vitro and in vivo studies. We evaluated uric acid levels in serum and urine, and xanthine oxidase (XOD) inhibition activity in the serum and liver tissue of a hyperuricemic rat model of potassium oxonate (PO)-induced hyperuricemic rats. In vitro study, XOD-inhibitory activity was the lowest among the test substances at the IC50 of ALP. However, the IC50 of DMLE-70 was significantly low compared with that of other DMLEs $(p<0.05)$. In PO-induced hyperuricemic rats, uric acid (UA) levels in serum and urine were significantly reduced in all DMLE-70 and allopurinol-treated (ALT) groups than in the PC group $(p<0.05)$. UA levels in urine were lower than those in serum in all DME groups. In PO-induced hyperuricemic rats, DMEE-200 reduced UA concentration in serum and increased UA excretion in the urine. These findings suggest that DMLE exerts antihyperuricemic and uricosuric effects on promoting UA excretion by enhanced secretion and inhibition of UA reabsorption in the kidneys. Thus, DMLE may be a potential treatment for hyperuricemia and gout.

\section{Introduction}

Hyperuricemia means elevated uric acid (UA) level (more than $6.8 \mathrm{mg} / \mathrm{dL}$ ) in the blood [1]. The disease is associated with a significantly increased risk of gout, cardiovascular disease, chronic kidney disease, and type 2 diabetes mellitus [2]. Serum UA (SUA) is the final product of purine metabolism [3]. Approximately two-thirds of SUA is produced from internal metabolic processes, and the rest is due to a high-purine diet [4]. Approximately $60 \%-70 \%$ of UA from the body is excreted through the kidneys, and the remaining is secreted in biliary secretions and the intestine. It is then further metabolized by gut bacteria in uricolysis [5]. Abnormal UA metabolism and decreased excretion by the kidneys are among the major causes of hyperuricemia [6].

Globally, hyperuricemia prevalence appears to be increasing as it is diagnosed in $5 \%-30 \%$ of the general population [7, 8]. It is also higher in men living in developed countries than women [9]. In the United States, the hyperuricemia prevalence rates are $20.2 \%$ in men and $20.0 \%$ in women [10]. In the Chinese rural population, the total estimated prevalence of hyperuricemia is $10.24 \%(12.80 \%$ in 
men and $8.56 \%$ in women) [11]. In the general Korean population, the age-standardized prevalence of hyperuricemia is $11.4 \%$ (17.0\% in men and $5.9 \%$ in women) [12]. The progressive increase of hyperuricemia worldwide may be linked to the rising prevalence of overweight and obesity and increased consumption of sugar-sweetened beverages, foods rich in purines, and alcohol [13].

As hyperuricemia results from increased production and decreased excretion, or both, of UA [14], it is crucial to prevent and treat the disorder to regulate the SUA level. UA is produced by xanthine oxidase (XOD), a rate-limiting enzyme that oxidizes hypoxanthine to xanthine, which is subsequently converted to UA [15]. Hence, SUA synthesis and concentration can be affected by XOD enzymatic activity [16]. Therefore, proteins involved in UA production and transport in the kidney may act as important drug targets for treating hyperuricemia.

XOD inhibitors (allopurinol (ALP) and febuxostat) and uricosuric agents (benzbromarone and probenecid) are presently used [17] to clinically treat hyperuricemia. However, these drugs are poorly tolerated and cause side effects, such as kidney diseases, hepatotoxicity, gastrointestinal symptoms, and hypersensitivity syndrome [18]. Therefore, more effective therapeutic agents for hyperuricemia with no adverse effects are needed. In previous studies, new therapeutic methods using herbs were offered to overcome these limitations of drugs for hyperuricemia [19].

Dendropanax morbifera H. Lév. (DM) is an evergreen broad-leaved tree of the Araliaceae family and is well known as a panacea and wild ginseng tree [9]. DM is an endemic species in Korea and is distributed in the country's southern regions [20]. In previous studies, extracts from roots and stems of DM have antioxidant [21], antibacterial [20], anticancer [22], antidiabetic [23], antiobesitic [9], antihyperglycemic [24], and antiatherogenic [25] properties. DM contains various bioactive compounds, such as triterpenoids, polyacetylene, phenolic substances, $\mathrm{L}_{\mathrm{L}}$-arginine, and $\gamma$-aminobutyric acid (GABA) [26]. $\mathrm{L}$-Arginine is a substrate of nitric oxide (NO) synthesis by NO synthase in almost all cell types. NO regulates glucose metabolism, fatty acids, and amino acids in mammals [27]. Additionally, GABA plays an essential physiological role in regulating cardiovascular function.

Therefore, in this study, the antioxidant and xanthine oxidase (XOD)-inhibitory activity of DM leaves (DML) was evaluated using different solvent extraction conditions (hot water and $30 \%, 50 \%$, and $70 \%$ ethanol). Additionally, the antihyperuricemic effects of DML extracts were investigated in a potassium oxonate ( $\mathrm{PO}$ )-induced hyperuricemia rat model, and the detailed mechanisms were explored.

\section{Materials and Methods}

2.1. Plant Extracts. DML was provided by Hyurim Hwangchil Co., Ltd. (Jinju, Korea), and dried in a shade, crushed, and stored in a refrigerator. The extracts were prepared as described by Kim et al. (2016) with some modifications. Approximately $100 \mathrm{~g}$ of the plant powder was extracted with $500 \mathrm{~mL}$ of water and $30 \%, 50 \%$, and $70 \%$ ethanol solution for $2 \mathrm{~h}$ at $80^{\circ} \mathrm{C}$. Subsequently, the solvent was evaporated using a rotary vacuum evaporator at $40^{\circ} \mathrm{C}$, freeze-dried, and weighted. Following the method above, the extraction was performed in triplicate for each solvent. The freeze-dried powders of DML ethanol extracts were named DMLEs and stored at $4^{\circ} \mathrm{C}$ until use.

2.2. Analysis of Chlorogenic Acid and Rutin Concentrations in $D M L E s$. The qualitative and quantitative analyses of chlorogenic acid (CGA) and rutin in DMLEs were conducted using an LC-MS 8050 chromatography system (Shimadzu, Kyoto, Japan) composed of a binary solvent delivery system (LC-30 AD), a controller (CBM 20A), an autosampler (SIL-30A), and a column thermostat (CTO20AC). Tandem mass spectrometry (MS/MS) analysis was performed positively and negatively on a triple quadrupole equipped with a positive electrospray ionization (+ESI) source. The optimal parameters of ESI-MS were as follows: interface temperature of $340^{\circ} \mathrm{C}$, DL temperature of $200^{\circ} \mathrm{C}$, nebulizing gas flow at $2.8 \mathrm{~L} / \mathrm{min}$, heating gas flow at $8 \mathrm{~L} / \mathrm{min}$, and temperature of drying gas at $400^{\circ} \mathrm{C}$. The active biological compounds were monitored using a scheduled multiple reaction monitoring mode and separated using a Kinetex C18 column $(150 \times 2.1 \mathrm{~mm}, 2.6 \mu \mathrm{m}$; Phenomenex, Torrance, CA, USA) at a flow rate of $0.3 \mathrm{~mL} / \mathrm{min}$, injection volume of $2 \mu \mathrm{L}$, and separation temperature of $40^{\circ} \mathrm{C}$. The mobile phase consisted of water containing $5 \mathrm{mM}$ ammonium acetate and $0.1 \%$ formic acid (A) and methanol containing $2.5 \mathrm{mM}$ ammonium acetate (B) (gradient $0-18 \mathrm{~min} ; 5 \%-100 \% \mathrm{~B}$ ).

2.3. 2,2-Diphenyl-1-picryl-hydrazyl (DPPH) Radical Scavenging Assay. The 2,2-diphenyl-1-picryl-hydrazyl (DPPH) radical scavenging assay was conducted following the description by Okoh et al. using vitamin $\mathrm{C}$ as the positive control [28]. Here, $0.1 \mathrm{~mL}$ of $0.135 \mathrm{mM}$ DPPH in methanol was mixed with $1.0 \mathrm{~mL}$ of the solution prepared in methanol containing $0.025-0.50 \mathrm{mg} / \mathrm{mL}$ of DMLEs, CGA, rutin, and vitamin $\mathrm{C}$. The reaction mixture was vortexed thoroughly, left in the dark at $25^{\circ} \mathrm{C}$ for $30 \mathrm{~min}$, and measured at $517 \mathrm{~nm}$ using a spectrophotometer. The DPPH radical scavenging effect was calculated using the equation below:

$$
\text { DPPH radical scavenging activity }(\%)=\left(1-\frac{\mathrm{A}_{\mathrm{s}}}{\mathrm{A}_{\mathrm{c}}}\right) \times 100 \text {. }
$$

Here, $A_{c}$ is the absorbance of the control reacted with methanol $(50 \mu \mathrm{L})$ and DPPH working solution $(1 \mathrm{~mL})$; and $A_{s}$ is the absorbance of the samples.

The sample concentration required for inhibiting 50\% DPPH radicals ( $\mathrm{IC}_{50} \mathrm{DPPH}$ values) was obtained by extrapolating the regression analysis. Antioxidant activity was evaluated based on this $\mathrm{IC}_{50}$ value.

2.4. Measurement of Reactive Oxygen Species (ROS) Production. The acute myelogenous leukemia cell line OCIAML-2 line from the Ontario Cancer Institute (Toronto, 
Canada) was cultured at $37^{\circ} \mathrm{C}$ in a $5 \% \mathrm{CO}_{2}$ atmosphere using minimum essential medium alpha ( $\alpha$-MEM) (Gibco BRL, Grand Island, NY, USA) with $10 \%$ heat-inactivated fetal bovine serum. The drug-resistant AML-2 sublines were selected from a parental cell line (AML-2/WT) after chronic exposure to doxorubicin. The cells were finally cultured in a fixed concentration $(100 \mathrm{ng} / \mathrm{mL})$ of doxorubicin. The AML2/DX100 cells were characterized by catalase downregulation and used to determine antioxidant effects [29]. The cells were seeded onto 96-well plates at a density of $1.5 \times 10^{4}$ cells per well in $100 \mu \mathrm{L}$ of growth media. At $50 \%$ confluence, cells were then loaded with $10 \mu \mathrm{M} 2^{\prime}, 7^{\prime}$ dichlorofluorescein diacetate (DCFH-DA; Sigma, St. Louis, $\mathrm{MO})$ and $4 \mathrm{mM}$ hydrogen peroxide $\left(\mathrm{H}_{2} \mathrm{O}_{2}\right.$; Sigma, St. Louis, $\mathrm{MO}$ ) in Hanks' balanced salt solution (HBSS; Gibco, Grand Island, NY, USA) at $37^{\circ} \mathrm{C}$ for $30 \mathrm{~min}$. After loading, cells were washed twice with $200 \mu \mathrm{L}$ of HBSS to remove excess fluorescent dye. Cells were then treated with 50 and $100 \mu \mathrm{g} /$ $\mathrm{mL}$ of DMLEs, CGA, rutin, and vitamin $\mathrm{C}$ and washed twice with $200 \mu \mathrm{L}$ of HBSS, and $100 \mu \mathrm{L}$ of HBSS/well was added. The fluorescence intensity of DCFH-DA was measured at an excitation wavelength of $485 \mathrm{~nm}$ and an emission wavelength of $520 \mathrm{~nm}$. Cells treated with $\mathrm{H}_{2} \mathrm{O}_{2}$ without DMLEs, CGA, rutin, and vitamin $\mathrm{C}$ were the controls. The measured fluorescence values were expressed as a percentage of the control.

2.5. Determination of XOD-Inhibitory Activity. The inhibitory effect on XOD was spectrophotometrically determined according to a method by Arimboor et al. with some modifications [30]. Briefly, $0.7 \mathrm{~mL}$ of 0.2 XOD unit in sodium phosphate buffer (0.1 M; pH 7.5) and $0.1 \mathrm{~mL}$ of various concentrations of DMLEs $(0.1-5.0 \mathrm{mg} / \mathrm{mL})$, CGA, rutin, and ALP were mixed at $37^{\circ} \mathrm{C}$ for $5 \mathrm{~min}$. The control did not contain test agents. After $5 \mathrm{~min}, 0.2 \mathrm{~mL}$ of $2 \mathrm{mM}$ xanthine in distilled water was added to the mixture. Each mixture was shaken at $37^{\circ} \mathrm{C}$ for $15 \mathrm{~min}$, and $1 \mathrm{~mL}$ of a stop buffer $(1 \mathrm{~N}$ $\mathrm{HCl}$ ) was added. ALP was used as a positive control. The absorbance of the mixture was measured at $290 \mathrm{~nm}$ using an ultraviolet-visible (UV-VIS) spectrophotometer (Optizen POP; CM Science, Busan, Korea). The $\mathrm{IC}_{50}$ values of the test samples and compounds were obtained using GraphPad Prism v.5.0 (GraphPad Software, Inc.).

2.6. Cell Cytotoxicity Assay. The human hepatocellular carcinoma cell line Hep3B (ATCC HB-8064) was grown in Dulbecco's modified Eagle's medium (DMEM; Merck KGaA, Darmstadt, Germany) supplemented with $10 \%$ (v/v) heat-inactivated fetal bovine serum (FBS), $100 \mathrm{U} / \mathrm{mL}$ penicillin, and $100 \mathrm{mg} / \mathrm{mL}$ streptomycin at $37^{\circ} \mathrm{C}$ under $5 \% \mathrm{CO}_{2}$. Cell viability assay was examined following the procedure described earlier [20]. Briefly, cells $\left(5 \times 10^{3}\right.$ cells/well) were plated onto a 96-well plate and cultured for $24 \mathrm{~h}$ at $37^{\circ} \mathrm{C}$ under $5 \% \mathrm{CO}_{2}$. After $24 \mathrm{~h}$, the cells were treated with various concentrations of DMLEs and incubated for $72 \mathrm{~h}$. After $72 \mathrm{~h}$, cells were washed with PBS, and $20 \mu \mathrm{L}$ of 3-(4,5-dimethylthiazol 2-yl)-2,5-diphenyltetrazolium bromide (MTT, $5 \mathrm{mg} /$ $\mathrm{mL}$ ) was added to each well. After incubation for $4 \mathrm{~h}$, dimethyl sulfoxide (DMSO) was added to dissolve formazan crystals from MTT reduction, and the amount of formazan salt was determined by measuring the optical density at $540 \mathrm{~nm}$ using an ELISA plate reader (Bio-Rad, Hercules, CA, USA). By comparing the absorbance of the wells of cells treated with different concentrations of DMLEs with the control, the viability of cells after treatment with DMLEs was calculated. The concentration of DMLEs that reduced the cell viability by $50 \%\left(\mathrm{IC}_{50}\right)$ was recorded.

2.7. Animals. Sixty Sprague-Dawley rats (male, 5 weeks, 110-150 g) were purchased from Orient Bio (Seongnam, Korea) and randomly distributed into different experimental groups. The rats were housed in polypropylene cages at an ambient temperature of $25^{\circ} \mathrm{C} \pm 1^{\circ} \mathrm{C}$ and $45 \%-55 \%$ relative humidity, with a $12: 12$-h light/dark cycle. Animals were provided with commercial food pellets and water ad libitum, unless stated otherwise. They were acclimatized to laboratory conditions for at least 1 week before experimenting on them. The experimental design was approved by the Institutional Animal Care and Use Committee of KemOn Inc. (Suwon, Korea) (Approval No. 2019-10-001), and all experiments were performed according to the guidelines established by the committee.

2.8. Induction of Hyperuricemia and Experimental Design. After acclimation for $7 \mathrm{~d}$, the uricase inhibitor PO was intraperitoneally injected into rats to induce hyperuricemia. To study the antihyperuricemic effects of the $70 \%$ ethanol extract from DML (DMLE-70), the rats were randomly divided into six groups ( $n=8$ per group): (1) a normal control group (NC), (2) a PO-induced hyperuricemia model group (PC), (3) a PC $+50 \mathrm{mg} / \mathrm{kg}$ ALP group (ALT), (4) a PC + $50 \mathrm{mg} / \mathrm{kg}$ DMLE-70 group (DMEE-50), (5) a PC $+100 \mathrm{mg} / \mathrm{kg}$ DMLE-70 group (DMEE-100), and (6) a PC $+200 \mathrm{mg} / \mathrm{kg}$ DMLE-70 group (DMEE-200). Rats in all groups, except for NC, were injected intraperitoneally with $250 \mathrm{mg} / \mathrm{kg}$ PO prepared in $0.5 \%$ carboxymethylcellulose (CMC) with $0.1 \mathrm{M}$ sodium acetate (pH 5.0) at $1,3,5$, and $7 \mathrm{~d}$ after acclimation, and NC was treated with $0.5 \%$ CMC with $0.1 \mathrm{M}$ sodium acetate. Rats of all groups, except for $\mathrm{NC}$ and PC, were administered extract once a day for $7 \mathrm{~d}$ from $1 \mathrm{~h}$ after the first injection of PO. The animals were anesthetized using isoflurane and sacrificed for sample collection.

2.9. Sample Collection and Analysis. Urine samples were collected during a 2 -h period using a metabolic cage following the last substance administration. The volume of urine collected was $4-6 \mathrm{~mL}$, and there was no difference in urine volume among the treated group. The samples were then centrifuged $\left(3000 \times \mathrm{g}\right.$ for $10 \mathrm{~min}$ at $\left.4^{\circ} \mathrm{C}\right)$ to remove particulate contaminants, and the supernatants were stored at $-80^{\circ} \mathrm{C}$ until analysis. After collecting urine samples, blood samples were collected via cardiac puncture and centrifuged $\left(3000 \times \mathrm{g}\right.$ for $10 \mathrm{~min}$ at $\left.4^{\circ} \mathrm{C}\right)$ to obtain serum. The separated serum was stored at $-80^{\circ} \mathrm{C}$ until analysis. According to the manufacturer's instructions, serum and urine levels of UA 
were determined using commercial assay kits (BioVision, Milpitas, CA, USA). At the end of the experiment, rats were euthanized by cervical dislocation after taking blood, and the liver tissue was collected and preserved at $-20^{\circ} \mathrm{C}$ to determine the XOD activity.

2.10. Statistical Analyses. Data are expressed as the mean \pm SEM. Statistical comparisons were analyzed by oneway analysis of variance (ANOVA) and Student's $t$-test using SPSS v.13.0. A p-value less than 0.05 was considered statistically significant.

\section{Results}

3.1. Extraction Yield. As a result of comparing the yield of DMLEs by the solvents, the yield of hot water extracts was highest at $24.14 \%$, and the extraction yield decreased as the ethanol content increased (30\% ethanol, $23.24 \%$; $50 \%$ ethanol, 21.02\%; and 70\% ethanol, 19.66\%).

3.2. Analysis of Chlorogenic Acid and Rutin Concentrations. Typical chromatograms of CGA and rutin are shown in Figure 1, representing the selected reaction monitoring chromatograms with a mass transition of CGA $(\mathrm{m} / z$ $355.00 \longrightarrow 145.15)$ and rutin $(m / z 615.00 \longrightarrow 465.10)$. No interference peaks were detected at the retention times of all analytes. CGA and rutin were eluted at retention times of 11.321 and $13.502 \mathrm{~min}$, respectively. In the LC-ESI-MS/MS analysis results, the concentrations of CGA and rutin increased depending on the ethanol concentration, and $70 \%$ DML ethanol extract (DMLE-70) had the highest CGA and rutin concentrations (Table 1 ).

3.3. Antioxidant Activity. DPPH radical scavenging activities were analyzed to determine the antioxidant activity of DMLEs. $\mathrm{IC}_{50}$ represents DPPH radical scavenging activities, and vitamin $\mathrm{C}$ was used as a positive control (Table 2). The $\mathrm{IC}_{50}$ of CGA was significantly low compared with that of DMLEs and rutin $(p<0.05)$. However, no significant difference in $\mathrm{IC}_{50}$ was observed between $\mathrm{CGA}$ and vitamin C. The $\mathrm{IC}_{50}$ of DMLE-70 was the most active among the DMLEs.

3.4. Measurement of ROS Production. The DCFH-DA assay was performed to evaluate the intracellular ROS scavenging activity of DMLEs and their active compounds in $\mathrm{H}_{2} \mathrm{O}_{2}$ treated AML-2/DX100 cells. After $400 \mu \mathrm{M} \mathrm{H}_{2} \mathrm{O}_{2}$ treatment, ROS scavenging activity was not significant among DMLEs, CGA, rutin, and vitamin $\mathrm{C}$ at a $50 \mu \mathrm{g} / \mathrm{mL}$ concentration. For treatment with $100 \mu \mathrm{g} / \mathrm{mL}$ of DMLEs or compounds, the $50 \%$ ethanol extract showed the strongest ROS scavenging activity. There was no significant difference between the ROS scavenging activity of the water extract and DMLE-30. By contrast, there was a significant difference among all test materials, except for DMLE-30 and DMLE-70 $(p<0.05)$ (Table 3).
3.5. Determination of XOD-Inhibitory Activity. DMLEs and compounds of the XOD-inhibitory activity are shown in Table 4 . The $\mathrm{IC}_{50}$ of ALP was $50 \mu \mathrm{g} / \mathrm{mL}$, which was the lowest among the test substances, followed by CGA. In addition, the $\mathrm{IC}_{50}$ of DMLE-70 was significantly low compared with that of other DMLEs $(p<0.05)$. However, the $\mathrm{IC}_{50}$ of rutin was more than $1000 \mu \mathrm{g} / \mathrm{mL}$, and there was almost no XODinhibitory activity.

3.6. Cell Cytotoxicity. In the MTT assay results, the $\mathrm{IC}_{50}$ values of DMLEs were more than $1.11 \mathrm{mg} / \mathrm{mL}$ (Table 5). The $\mathrm{IC}_{50}$ value of DMLW $(2.08 \mathrm{mg} / \mathrm{mL})$ was significantly high compared with that of other DMLEs $(p<0.05)$. In the ethanol extracts, the $\mathrm{IC}_{50}$ value decreased with an increase in the percentage of ethanol in the extraction solvents. However, no statistically significant difference was observed between each ethanol extract. Therefore, it was confirmed that the $\mathrm{IC}_{50}$ values of all DMLEs were more than $1.11 \mathrm{mg} / \mathrm{mL}$, indicating almost no cytotoxicity.

3.7. UA Levels in Serum and Urine in the Hyperuricemic Rat Model. The PC group exhibited significantly higher UA levels in serum and urine than the NC group did $(p<0.05)$, indicating that hyperuricemia was effectively established. UA levels in serum and urine were significantly reduced in all DMLE-70 and ALT groups compared with the PC group $(p<0.05)$ (Figure 2). In all DME groups, UA levels in urine were lower than those in serum. In PO-induced hyperuricemic rats, DMEE-200 reduced UA concentration in serum and increased UA excretion through the urine.

3.8. XOD Activity in Serum and Liver Tissue. XOD activity in serum showed no significant differences between all groups, except for the ALT group (Table 6). There was no significant difference in hepatic XOD activity between the PO-induced hyperuricemic rat group (PC) and the groups treated with DMLE-70 at concentrations of 50 and $100 \mathrm{mg} / \mathrm{kg}$. However, hepatic XOD activity in the PC group was significantly increased compared with that in the group treated with DMLE-70 at a $200 \mathrm{mg} / \mathrm{kg}(p<0.05)$ concentration. Meanwhile, treatment with ALP significantly reduced XOD activity in serum and liver tissue compared with that in all groups $(p<0.05)$.

\section{Discussion}

Hyperuricemia is a metabolic disorder characterized by a high UA level in serum due to an imbalance between UA production and excretion and causes gout [14]. Recently, the prevalence of hyperuricemia has continuously increased worldwide [31]. Currently, there are a limited number of drugs available for treating hyperuricemia, and many of these drugs have side effects.

Among the uricosuric agents, benzbromarone has been discontinued since 2003 because of severe hepatotoxicity reported in most European countries [32]. Although ALP belonging to $\mathrm{XO}$ inhibitors has been mainly used to treat 


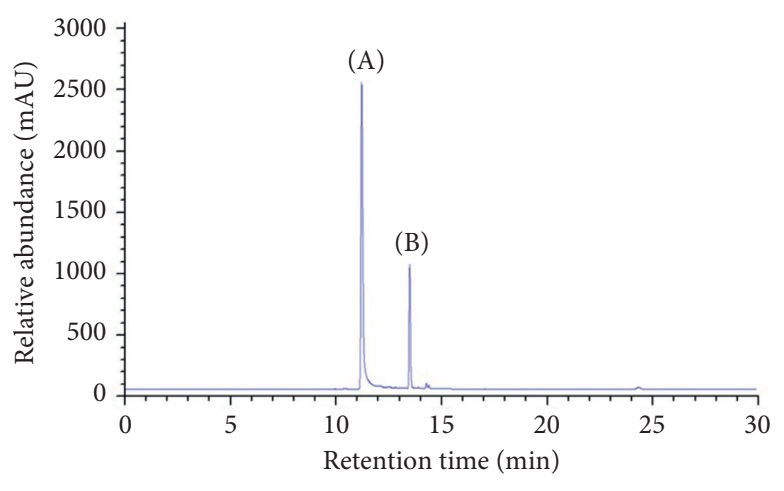

(A)

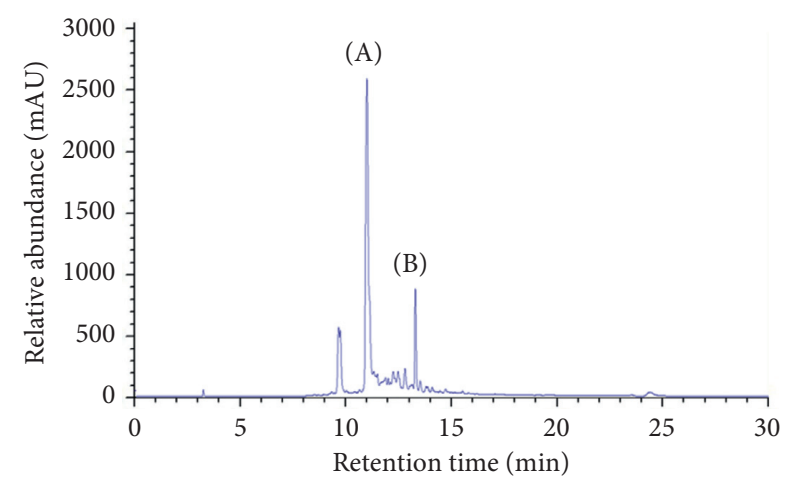

(B)

FIgURE 1: Representative liquid chromatogram of (A) standard and (B) Dendropanax morbifera leaf 70\% ethanol extract.

TAвLE 1: Chlorogenic acid and rutin contents in Dendropanax morbifera leaf extracts by water and various ethanol concentrations.

\begin{tabular}{lcc}
\hline Compounds & Chlorogenic acid (mg/g) & Rutin $(\mathrm{mg} / \mathrm{g})$ \\
\hline DMLW & $6.42 \pm 0.02^{\mathrm{a}}$ & $10.39 \pm 0.06^{\mathrm{a}}$ \\
DMLE-30 & $13.25 \pm 0.24^{\mathrm{b}}$ & $16.39 \pm 0.22^{\mathrm{b}}$ \\
DMLE-50 & $17.42 \pm 0.30^{\mathrm{c}}$ & $23.74 \pm 0.31^{\mathrm{c}}$ \\
DMLE-70 & $21.46 \pm 0.12^{\mathrm{d}}$ & $29.52 \pm 0.13^{\mathrm{d}}$ \\
\hline
\end{tabular}

DMLW, water extract of Dendropanax morbifera leaf; DMLE-30, 30\% ethanol extract of Dendropanax morbifera leaf; DMLE-50, 50\% ethanol extract of Dendropanax morbifera leaf; DMLE-70, 70\% ethanol extract of Dendropanax morbifera leaf. ${ }^{\mathrm{a}, \mathrm{b}, \mathrm{c}, \mathrm{d}}$ Mean values with different superscript letters in the same row show significant differences at $p<0.05$ by one-way ANOVA.

TABLE 2: 2,2-diphenyl-1-picryl-hydrazyl radical scavenging ability of Dendropanax morbifera leaf extracts and its active compounds.

\begin{tabular}{lcc}
\hline Compounds & $\mathrm{IC}_{50}(\mu \mathrm{g} / \mathrm{mL})^{1}$ & ${\text { Relative activity }(\%)^{2}}^{\mathrm{a}}$ \\
\hline DMLW & $102.53 \pm 3.59^{\mathrm{a}}$ & 5.36 \\
DMLE-30 & $89.23 \pm 2.36^{\mathrm{b}}$ & 6.16 \\
DMLE-50 & $85.13 \pm 3.13^{\mathrm{b}}$ & 6.46 \\
DMLE-70 & $78.93 \pm 0.58^{\mathrm{c}}$ & 6.97 \\
CGA & $3.94 \pm 0.05^{\mathrm{d}}$ & 139.48 \\
Rutin & $37.60 \pm 342^{\mathrm{e}}$ & 14.63 \\
Vitamin C & $5.50 \pm 0.40^{\mathrm{d}}$ & 100.00 \\
\hline
\end{tabular}

DMLW, water extract of Dendropanax morbifera leaf; DMLE-30, 30\% ethanol extract of Dendropanax morbifera leaf; DMLE-50, 50\% ethanol extract of Dendropanax morbifera leaf; DMLE-70, 70\% ethanol extract of Dendropanax morbifera leaf; CGA, chlorogenic acid. ${ }^{1} \mathrm{IC}_{50}$ value is a concentration of each sample for scavenging activity of $50 \%$ DPPH radical. ${ }^{2}$ A ratio of $\mathrm{IC}_{50}$ value compared with vitamin $\mathrm{C}$ used as a positive control. ${ }^{\mathrm{a}, \mathrm{b}, \mathrm{c}, \mathrm{d}}$ Mean values with different superscript letters in the same row show significant differences at $p<0.05$ by one-way ANOVA.

TABLE 3: Reactive oxygen species (ROS) scavenging ability of Dendropanax morbifera leaf extracts and its active compounds.

\begin{tabular}{lcr}
\hline Compounds & \multicolumn{1}{c}{ ROS scavenging abilities (\%) } \\
\hline DMLW & $50 \mu \mathrm{g} / \mathrm{mL}$ & $78.19 \pm 2.43^{\mathrm{a}}$ \\
DMLE-30 & $42.74 \pm 0.96$ & $80.92 \pm 3.01^{\mathrm{ab}}$ \\
DMLE-50 & $43.22 \pm 1.75$ & $84.86 \pm 2.63^{\mathrm{b}}$ \\
DMLE-70 & $44.58 \pm 2.82$ & $57.14 \pm 6.13^{\mathrm{c}}$ \\
CGA & $44.49 \pm 3.31$ & $64.85 \pm 3.31^{\mathrm{d}}$ \\
Rutin & $41.67 \pm 3.39$ & $66.37 \pm 2.72^{\mathrm{d}}$ \\
Vitamin C & $44.58 \pm 2.14$ & $56.53 \pm 4.08^{\mathrm{c}}$ \\
\hline
\end{tabular}

DMLW, water extract of Dendropanax morbifera leaf; DMLE-30, 30\% ethanol extract of Dendropanax morbifera leaf; DMLE-50, 50\% ethanol extract of Dendropanax morbifera leaf; DMLE-70, 70\% ethanol extract of Dendropanax morbifera leaf; CGA, chlorogenic acid. ${ }^{\mathrm{a}, \mathrm{b}, \mathrm{c}, \mathrm{d}}$ Mean values with different superscript letters in the same row show significant differences at $p<0.05$ by one-way ANOVA. 
TABLE 4: Xanthine oxidase inhibition activity of Dendropanax morbifera leaf extracts and its active compounds.

\begin{tabular}{lcc}
\hline Compounds & $\mathrm{IC}_{50}(\mu \mathrm{g} / \mathrm{mL})^{1}$ & Relative activity $(\%)^{2}$ \\
\hline DMLW & $527.07 \pm 10.25^{\mathrm{a}}$ & 1.95 \\
DMLE-30 & $441.90 \pm 5.15^{\mathrm{b}}$ & 2.32 \\
DMLE-50 & $324.29 \pm 1.08^{\mathrm{c}}$ & 3.16 \\
DMLE-70 & $216.98 \pm 7.41^{\mathrm{d}}$ & 4.73 \\
CGA & $69.07 \pm 2.85^{\mathrm{e}}$ & 14.85 \\
Rutin & $>1000$ & -3 \\
ALP & $10.25 \pm 0.26^{\mathrm{f}}$ & 100.00 \\
\hline
\end{tabular}

DMLW, water extract of Dendropanax morbifera leaf; DMLE-30, 30\% ethanol extract of Dendropanax morbifera leaf; DMLE-50, 50\% ethanol extract of Dendropanax morbifera leaf; DMLE-70, 70\% ethanol extract of Dendropanax morbifera leaf; CGA, chlorogenic acid; ALP, allopurinol. ${ }^{1}$ The concentration of each compound needed to inhibit $50 \%$ of xanthine oxidase activity. ${ }^{2} \mathrm{~A}$ ratio of $\mathrm{IC}_{50}$ value compared with that of allopurinol. ${ }^{3}$ Not identified. ${ }^{\mathrm{a}, \mathrm{b}, \mathrm{c}, \mathrm{d}} \mathrm{Mean}$ values with different superscript letters in the same row show significant differences at $p<0.05$ by one-way ANOVA.

TABle 5: Half maximal inhibitory concentration $\left(\mathrm{IC}_{50}\right)$ of Dendropanax morbifera leaf extracts in the Hep3B cell lines after $72 \mathrm{~h}$ of exposure.

\begin{tabular}{lc}
\hline Compounds & $\mathrm{IC}_{50}(\mathrm{mg} / \mathrm{mL})$ \\
\hline DMLW & $2.08 \pm 0.16^{\mathrm{a}}$ \\
DMLE-30 & $1.23 \pm 0.01^{\mathrm{b}}$ \\
DMLE-50 & $1.19 \pm 0.02^{\mathrm{b}}$ \\
DMLE-70 & $1.11 \pm 0.01^{\mathrm{b}}$ \\
\hline
\end{tabular}

DMLW, water extract of Dendropanax morbifera leaf; DMLE-30, 30\% ethanol extract of Dendropanax morbifera leaf; DMLE-50, 50\% ethanol extract of Dendropanax morbifera leaf; DMLE-70, 70\% ethanol extract of Dendropanax morbifera leaf. ${ }^{\mathrm{a}, \mathrm{b}, \mathrm{c}, \mathrm{d}}$ Mean values with different superscript letters in the same row show significant differences at $p<0.05$ by one-way ANOVA.

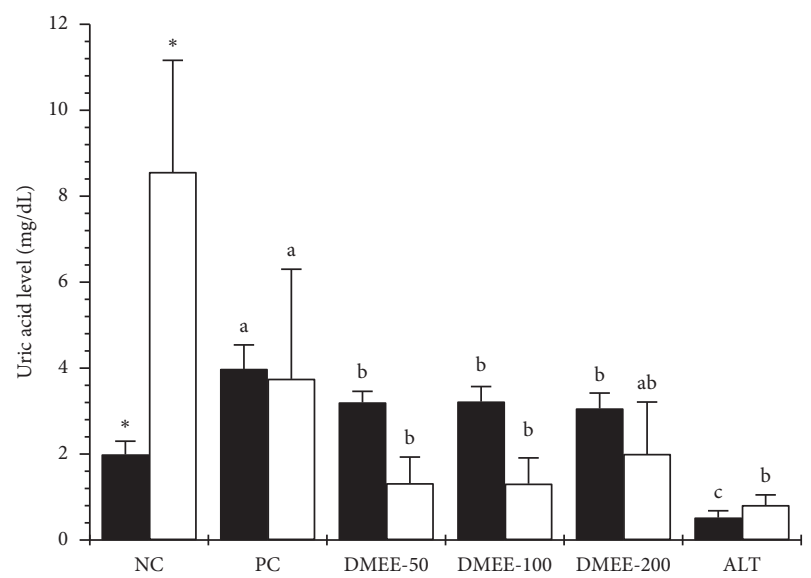

Figure 2: Effects of $70 \%$ ethanol extracts from Dendropanax morbifera leaf (DMLE-70) on serum (ם) and urine uric acid ( $\square$ ) levels in rats with potassium oxonate-induced hyperuricemia. NC, normal control group; PC, PO-induced hyperuricemia model group; DMEE-50, PC + 50 mg/kg DMLE-70 group; DMEE-100, PC + $100 \mathrm{mg} / \mathrm{kg}$ DMLE-70 group; DMEE-200, PC + 200 mg/kg DMLE70 group; ALT, PC $+50 \mathrm{mg} / \mathrm{kg}$ allopurinol group. ${ }^{*}$, significantly different from PC, $p<0.05$. a, b, c, d: Mean values with different superscript letters in the same row show significant differences at $p<0.05$ by one-way ANOVA.

hyperuricemia, this drug has been associated with severe cutaneous adverse reactions [33]. Therefore, many previous studies have been conducted to develop safe therapeutic agents using natural products for hyperuricemia without side effects $[14,19,30]$.

This study showed the antioxidant, XOD-inhibitory, and antihyperuricemic activities of DMLEs and determined active components. The upregulation of XOD expression and activity increases reactive oxygen species (ROS) production. The XOD activity produces superoxide anions that can rapidly respond with nitric oxide to form the cytotoxic oxidant leading to various diseases including endothelial dysfunction and cardiovascular diseases [34]. When the local levels of ROS are increasing, they cause considerable cellular damage and generate other more reactive radicals [35]. As regards the production of ROS by $\mathrm{XO}$, under conditions of oxidative stress, $\mathrm{XO}$ activity prevails to $\mathrm{XDH}$ activity, resulting in further ROS production. In cultures of endothelial cells, NOX maintains $\mathrm{XO}$ levels and $\mathrm{XO}$ is responsible for increased ROS production [36]. In this study, DMLE-70 had the strongest DPPH free radical scavenging activity and XOD-inhibitory activity among the DMLEs (Tables 2-4). These findings suggest that DMLEs inhibit hyperuricemia by inhibiting the generation of ROS through its XOD-inhibitory effect. However, DMLE-50 showed the strongest ROS scavenging activity at a $100 \mu \mathrm{g} / \mathrm{mL}$ concentration among the DMLEs. On the basis of these results, DMLE-70 was selected for further in vivo experiments.

In the LC-MS/MS analysis results, DMLE-70 had the highest CGA and rutin concentrations (Table 1). CGA is an abundant polyphenol in human diet and Chinese medicines and possesses extensive pharmacological antimicrobial, antioxidant, anti-inflammatory, protection of cardiovascular and cerebrovascular system [37], and antigout activities [38]. Rutin is an essential flavonoid in herbal foods, which significantly reduced SUA levels in mice with hyperuricemia [39] and increased urinary UA concentration in rats with renal dysfunction [40]. In a previous study [41], the CGA and rutin contents from DML extracts in $70 \%$ ethanol for $2 \mathrm{~h}$ were the highest at 12.33 and $14.09 \mathrm{mg} / \mathrm{g}$, respectively. In another study, the CGA concentration in the $30 \%$ DML ethanol extract collected in November was the highest at $36.55 \mathrm{mg} / \mathrm{g}$, and the rutin content in the $60 \%$ DML ethanol extract collected in May was the highest at $116.71 \mathrm{mg} / \mathrm{g}$ [42]. In this study, the concentrations of CGA and rutin were approximately 0.5 and 0.25 times lower than those of Youn et al. but twice 
TABLE 6: Xanthine oxidase activity of Dendropanax morbifera leaf extracts in serum and liver tissue of the hyperuricemic rat model.

\begin{tabular}{lcc}
\hline Compounds & Serum $(\mathrm{mg} / \mathrm{dL})$ & Liver $(\mathrm{mU} / \mathrm{mg}$ protein $)$ \\
\hline NC & $1.03 \pm 0.05^{\mathrm{a}}$ & $0.049 \pm 0.003^{\mathrm{a}}$ \\
PC & $0.96 \pm 0.06^{\mathrm{a}}$ & $0.046 \pm 0.005^{\mathrm{ab}}$ \\
DMEE-50 & $1.00 \pm 0.04^{\mathrm{a}}$ & $0.043 \pm 0.002^{\mathrm{b}}$ \\
DMEE-100 & $0.94 \pm 0.03^{\mathrm{a}}$ & $0.043 \pm 0.002^{\mathrm{b}}$ \\
DMEE-200 & $0.94 \pm 0.07^{\mathrm{a}}$ & $0.037 \pm 0.002^{\mathrm{c}}$ \\
Allopurinol & $0.35 \pm 0.01^{\mathrm{b}}$ & $0.024 \pm 0.001^{\mathrm{d}}$ \\
\hline
\end{tabular}

NC, normal control group; PC, PO-induced hyperuricemia model group; DMEE-50, PC + $50 \mathrm{mg} / \mathrm{kg}$ DMLE-70 group; DMEE-100, PC $+100 \mathrm{mg} / \mathrm{kg}$ DMLE-70 group; DMEE-200, PC $+200 \mathrm{mg} / \mathrm{kg}$ DMLE-70 group. ${ }^{\mathrm{a}, \mathrm{b}, \mathrm{c}, \mathrm{d}}$ Mean values with different superscript letters in the same row show significant differences at $p<0.05$ by one-way ANOVA.

as high as those of Hwang et al. [41] (Table 1). This showed that the difference in CGA content and rutin depends on the harvesting location, season, and extraction conditions of DML [42].

We investigated whether DMLE extract showed antihyperuricemic effects in PO-induced hyperuricemic rats. In this study, DMEE-50, 100, and 200 significantly decreased serum UA levels compared with the PC group $(p<0.05)$. Additionally, DMEE-50 and 100 reduced UA levels in urine compared with the PC group $(p<0.05)$. Meanwhile, the urine UA levels of DMEE-200 were higher than those of DMEE-50 and 100, although there were no significant differences in urine UA levels between DMEE-200 and DMEE-50 and 100. These results suggest that DMEE-200 alleviates hyperuricemia by decreasing the UA concentration in the blood and increasing UA excretion in the urine (Figure 2).

Additionally, we demonstrated that DMLE could decrease serum UA levels in PO-induced hyperuricemic mice by inhibiting hepatic XOD activity. Inhibiting XOD activity in the liver reduces UA production and enhances UA excretion $[43,44]$. DMLE-70 at all doses did not exhibit potent hypouricemic effects in serum compared with PC, but inhibiting hepatic XOD activity was significantly effective with DMEE-200 compared with the PC (Table 6), although the amount of uric acid excreted in urine was decreased compared with PC. These results elucidated that the hypouricemic effect of DMLE is caused by inhibiting XOD, which is a crucial enzyme in the biosynthetic pathway of UA. The effect of DMLE on renal transporters, which contribute to UA reabsorption, is unknown and will be the focus of our subsequent study.

This study demonstrated that DMLE reduced serum UA levels and enhanced the excretion of UA in rats with POinduced hyperuricemia. DMLE has an antioxidant activity in vitro and inhibits $\mathrm{XO}$ activity of the liver and serum in vivo, thus preventing hyperuricemia. This study elucidates that DMLE exhibits beneficial effects and may be potentially helpful in treating hyperuricemia and gout.

\section{Data Availability}

The data are available from the corresponding author upon request.

\section{Conflicts of Interest}

The authors have no conflicts of interest to declare with regard to the manuscript.

\section{Authors' Contributions}

KI Park conceived and designed the study and supported all materials. DH Lee and JK Kim performed the experiments, treatments/animal care, and statistical analysis. YJ Han wrote the manuscript. KI Park reviewed the literature, revised the manuscript, and coordinated the study. DH Lee and JK Kim equally contributed to this work.

\section{References}

[1] S.-K. Kim, "Interrelationship of uric acid, gout, and metabolic syndrome: focus on hypertension, cardiovascular disease, and insulin resistance," Journal of Rheumatic Diseases, vol. 25, no. 1, pp. 19-27, 2018.

[2] Q. Ni, X. Lu, C. Chen, H. Du, and R. Zhang, "Risk factors for the development of hyperuricemia: a STROBE-compliant cross-sectional and longitudinal study," Medicine, vol. 98, no. 42, p. e17597, 2019.

[3] G. Glantzounis, E. Tsimoyiannis, A. Kappas, and D. Galaris, "Uric acid and oxidative stress," Current Pharmaceutical Design, vol. 11, no. 32, pp. 4145-4151, 2005.

[4] E. S. Perez, M. A. G. Medina, M. L. Lomeli et al., "Association between serum uric acid and metabolic syndrome components in prepubertal obese children (tanner stage I) from Nuevo Leon, Mexico-a preliminary study," BMC Obesity, vol. 4, no. 25, 2017.

[5] I. A. Bobulescu and O. W. Moe, "Renal transport of uric acid: evolving concepts and uncertainties," Advances in Chronic Kidney Disease, vol. 19, no. 6, pp. 358-371, 2012.

[6] D. J. Stewart, V. Langlois, and D. Noone, "Hyperuricemia and hypertension: links and risks," Integrated Blood Pressure Control, vol. 12, pp. 43-62, 2019.

[7] A. F. G. Cicero, M. Rosticci, M. Bove et al., "Serum uric acid change and modification of blood pressure and fasting plasma glucose in an overall healthy population sample: data from the Brisighella heart study," Annals of Medicine, vol. 49, no. 4, pp. 275-282, 2017.

[8] T. Katova, I. Simova, Y. Yotov, K. Peeva, and N. Georgieva, "Hyperuricemia and cardiovascular risk in the Bulgarian population," Cureus, vol. 1, p. 101, 2020.

[9] P. Song, H. Wang, W. Xia, X. Chang, M. Wang, and L. An, "Prevalence and correlates of hyperuricemia in the middleaged and older adults in China," Scientific Reports, vol. 8, p. 4314, 2018.

[10] M. Chen-Xu, C. Yokose, S. K. Rai, M. H. Pillinger, and H. K. Choi, "Contemporary prevalence of gout and hyperuricemia in the United States and decadal trends: the national health and nutrition examination survey, 2007-2016," Arthritis \& Rheumatology, vol. 71, no. 6, pp. 991-999, 2019.

[11] X. Dong, H. Zhang, F. Wang et al., "Epidemiology and prevalence of hyperuricemia among men and women in Chinese rural population: the Henan rural cohort study," Modern Rheumatology, vol. 30, no. 5, pp. 910-920, 2020.

[12] Y. Kim, J. Kang, and G.-T. Kim, "Prevalence of hyperuricemia and its associated factors in the general Korean population: an analysis of a population-based nationally representative 
sample," Clinical Rheumatology, vol. 37, no. 9, pp. 2529-2538, 2018.

[13] G. Desideri, J. G. Puig, and P. Richette, "The management of hyperuricemia with urate deposition," Current Medical Research and Opinion, vol. 31, no. 2, pp. 27-32, 2015.

[14] Y. S. Lee, E. Son, S. H. Kim, Y. M. Lee, O. S. Kim, and D. S. Kim, "Synergistic uric acid-lowering effects of the combination of Chrysanthemum indicum linne flower and cinnamomum cassia (L.) J. Persl bark extracts," Evidencebased Complementary and Alternative Medicine: ECAM, vol. 2017, Article ID 9764843, 9 pages, 2017.

[15] Y. Zhang, L. Jin, J. Liu et al., "Effect and mechanism of dioscin from Dioscorea spongiosa on uric acid excretion in animal model of hyperuricemia," Journal of Ethnopharmacology, vol. 214, pp. 29-36, 2018.

[16] L.-L. Kang, D.-M. Zhang, C.-H. Ma et al., "Cinnamaldehyde and allopurinol reduce fructose-induced cardiac inflammation and fibrosis by attenuating CD36-mediated TLR4/6IRAK4/1 signaling to suppress NLRP3 inflammasome activation," Scientific Reports, vol. 6, no. 1, p. 27460, 2016.

[17] D.-R. Oh, J. R. Kim, C. Y. Choi et al., "Effects of ChondroT on potassium Oxonate-induced Hyperuricemic mice: downregulation of xanthine oxidase and urate transporter 1," $B M C$ Complementary and Alternative Medicine, vol. 19, no. 1, p. 10, 2019.

[18] R.-J. Chen, M.-H. Chen, Y.-L. Chen et al., "Evaluating the urate-lowering effects of different microbial fermented extracts in hyperuricemic models accompanied with a safety study," Journal of Food and Drug Analysis, vol. 25, no. 3, pp. 597-606, 2017.

[19] R. Bao, M. Liu, D. Wang et al., "Effect of eurycoma longifolia stem extract on uric acid excretion in hyperuricemia mice," Frontiers in Pharmacology, vol. 10, p. 1464, 2019.

[20] R.-W. Kim, S.-Y. Lee, S.-G. Kim, Y.-R. Heo, and M.-K. Son, "Antimicrobial, antioxidant and cytotoxic activities of Dendropanax morbifera Léveille extract for mouthwash and denture cleaning solution," The Journal of Advanced Prosthodontics, vol. 8, no. 3, pp. 172-180, 2016.

[21] W. Kim, D. W. Kim, D. Y. Yoo et al., "Antioxidant effects of Dendropanax morbifera Leveille extract in the hippocampus of mercury-exposed rats," BMC Complementary and Alternative Medicine, vol. 15, 2015.

[22] T. K. Hyun, M.-o. Kim, H. Lee, Y. Kim, E. Kim, and J.-S. Kim, "Evaluation of anti-oxidant and anti-cancer properties of Dendropanax morbifera Léveille," Food Chemistry, vol. 141, no. 3, pp. 1947-1955, 2013.

[23] H.-I. Moon, "Antidiabetic effects of dendropanoxide from leaves of Dendropanax morbifera Leveille in normal and streptozotocin-induced diabetic rats," Human \& Experimental Toxicology, vol. 30, no. 8, pp. 870-875, 2011.

[24] X. Tan and H. K. Ryu, "Effects of Dendropanax morbifera leaf extracts on lipid profiles in mice fed a high-fat and highcholesterol diet," Journal of the Korean Society of Food Science and Nutrition, vol. 44, no. 5, pp. 641-648, 2015.

[25] I. M. Chung, M. Y. Kim, W. H. Park, and H. I. Moon, "Antiatherogenic activity of Dendropanax morbifera essential oil in rats," Die Pharmazie, vol. 64, no. 8, pp. 547-549, 2009.

[26] E. J. Rupa, L. Arunkumar, Y. Han et al., "Dendropanax morbifera extract-mediated $\mathrm{ZnO}$ nanoparticles loaded with indole-3-carbinol for enhancement of anticancer efficacy in the A549 human lung carcinoma cell line," Materials (Basel, Switzerland), vol. 13, no. 14, 2020.

[27] S. Park, K. H. Lee, W. S. Kang, J. S. Kim, and S. Kim, "Endothelium-dependent and endothelium-independent vasorelaxant effects of unripe Rubus coreanus Miq. and Dendropanax morbiferus $\mathrm{H}$. Lév. extracts on rat aortic rings," BMC Complementary Medicine and Therapies, vol. 20, no. 1, p. 190, 2020.

[28] S. Okoh, O. Asekun, O. Familoni, and A. Afolayan, "Antioxidant and free radical scavenging capacity of seed and shell essential oils extracted from Abrus precatorius (L)," Antioxidants, vol. 3, no. 2, pp. 278-287, 2014.

[29] J.-M. Jeong, C.-H. Choi, S.-K. Kang, I.-H. Lee, J.-Y. Lee, and H. Jung, "Antioxidant and chemosensitizing effects of flavonoids with hydroxy and/or methoxy groups and structureactivity relationship," Journal of Pharmacy \& Pharmaceutical Sciences, vol. 10, no. 4, pp. 537-546, 2007.

[30] R. Arimboor, M. Rangan, S. G. Aravind, and C. Arumughan, "Tetrahydroamentoflavone (THA) from Semecarpus anacardium as a potent inhibitor of xanthine oxidase," Journal of Ethnopharmacology, vol. 133, no. 3, pp. 1117-1120, 2011.

[31] E. Zitt, A. Fischer, K. Lhotta, H. Concin, and G. Nagel, "Sexand age-specific variations, temporal trends and metabolic determinants of serum uric acid concentrations in a large population-based Austrian cohort," Scientific Reports, vol. 10, no. 1, p. 7578, 2020.

[32] T. L. Jansen, M. K. Reinders, E. N. Van Roon, and J. R. Brouwers, "Benzbromarone withdrawn from the European market: another case of "absence of evidence is evidence of absence"?" Clinical and Experimental Rheumatology, vol. 22, no. 5, p. 651, 2004.

[33] S. N. Ramasamy, C. S. Korb-Wells, D. R. Kannangara et al., "Allopurinol hypersensitivity: a systematic review of all published cases, 1950-2012,” Drug Safety, vol. 36, no. 10, pp. 953-980, 2013.

[34] M. Gliozzi, N. Malara, S. Muscoli, and V. Mollace, "The treatment of hyperuricemia," International Journal of Cardiology, vol. 213, pp. 23-27, 2013.

[35] C. E. Berry and J. M. Hare, "Xanthine oxidoreductase and cardiovascular disease: molecular mechanisms and pathophysiological implications," The Journal of Physiology, vol. 555, no. Pt 3, pp. 589-606, 2004.

[36] N. Liu, H. Xu, Q. Sun et al., "The role of oxidative stress in hyperuricemia and xanthine oxidoreductase (XOR) inhibitors," Oxidative Medicine and Cellular Longevity, vol. 2021, Article ID 1470380, 15 pages, 2021.

[37] L.-M. Liu, S.-F. Cheng, P.-C. Shieh et al., "The methanol extract of Euonymus laxiflorus, Rubia lanceolata and Gardenia jasminoides inhibits xanthine oxidase and reduce serum uric acid level in rats," Food and Chemical Toxicology, vol. 70, pp. 179-184, 2014.

[38] Z. Q. Meng, Z. H. Tang, Y. X. Yan et al., "Study on the antigout activity of chlorogenic acid: improvement on hyperuricemia and gouty inflammation," The American Journal of Chinese Medicine, vol. 42, no. 6, pp. 1471-1483, 2014.

[39] Y. S. Chen, Q. H. Hu, X. Zhang, Q. Zhu, and L. D. Kong, "Beneficial effect of rutin on oxonate-induced hyperuricemia and renal dysfunction in mice," Pharmacology, vol. 92, no. 1-2, pp. 75-83, 2013.

[40] Q.-H. Hu, C. Wang, J.-M. Li, D.-M. Zhang, and L.-D. Kong, "Allopurinol, rutin, and quercetin attenuate hyperuricemia and renal dysfunction in rats induced by fructose intake: renal organic ion transporter involvement," American Journal of Physiology-Renal Physiology, vol. 297, no. 4, pp. F1080-F1091, 2009.

[41] C. E. Hwang, S. C. Kim, C. S. Cho, W. Y. Song, O. S. Joo, and K. M. Cho, "Comparison of chlorogenic acid and rutin contents and antioxidant activity of Dendropanax morbiferus 
extracts according to ethanol concentration," Korean Journal of Food Preservation, vol. 27, no. 7, pp. 880-887, 2020.

[42] J. S. Youn, Y.-J. Kim, H. J. Na et al., "Antioxidant activity and contents of leaf extracts obtained from Dendropanax morbifera LEV are dependent on the collecting season and extraction conditions," Food Science and Biotechnology, vol. 28, no. 1, pp. 201-207, 2019.

[43] Y. Chen, Z. Zhao, Y. Li et al., "Baicalein alleviates hyperuricemia by promoting uric acid excretion and inhibiting xanthine oxidase," Phytomedicine, vol. 80, Article ID 153374, 2021.

[44] L. L. Jiang, X. Gong, M. Y. Ji, C. C. Wang, J. H. Wang, and M. H. Li, "Bioactive compounds from plant-based functional foods: a promising choice for the prevention and management of hyperuricemia," Foods (Basel, Switzerland), vol. 9, no. 8, 2020 . 